\title{
Acrodermatitis Enteropathica
}

National Cancer Institute

\section{Source}

National Cancer Institute. Acrodermatitis Enteropathica. NCI Thesaurus. Code C128802.

An autosomal recessive genetic disorder caused by mutations in the SLC39A4 gene,

encoding zinc transporter ZIP4. The condition is characterized by zinc deficiency,

periorificial and acral dermatitis, and diarrhea. 